\title{
Estimation of canopy attributes of wild cacao trees using digital cover photography and machine learning algorithms
}

\author{
Julio Martin Duarte-Carvajalino ${ }^{(1)}$, \\ Mónica Paramo-Alvarez ${ }^{(2)}$, \\ Pablo Fernando Ramos-Calderón ${ }^{(3)}$, \\ Carlos Eduardo González-Orozco ${ }^{(4)}$
}

(1) Corporación Colombiana de Investigación Agropecuaria - AGROSAVIA, Centro de Investigación Tibaitatá, Km 14 vía Mosquera, Bogotá, Cundinamarca (Colombia); (2) Corporación Colombiana de Investigación Agropecuaria - AGROSAVIA, Sede Central, Km 14 vía Mosquera, Bogotá (Colombia); (3) Corporación Colombiana de Investigación Agropecuaria - AGROSAVIA, Centro de Investigación Nataima, Km 9 vía Espinal-Chicoral, Tolima, Sede Florencia, Caquetá (Colombia); (4) Corporación Colombiana de Investigación Agropecuaria - AGROSAVIA, Centro de Investigación La Libertad, Km 14 vía Villavicencio, Puerto López, Meta (Colombia)

@ Julio Martin Duarte-Carvajalino (jmduarte@agrosavia.co)

Received: Jul 23, 2021 - Accepted: Sep 08, 2021

Citation: Duarte-Carvajalino JM, ParamoAlvarez M, Ramos-Calderón PF, GonzalezOrozco CE (2021). Estimation of canopy attributes of wild cacao trees using digital cover photography and machine learning algorithms. iForest 14: 517-521. - doi: 10.3832/ifor3936-014 [online 2021-11-17]

Communicated by: Nicola Puletti
Surveying canopy attributes while conducting fieldwork in the rain forest is time-consuming. Low-cost imagery such as digital cover photography is a potential source of information to speed up the process of vegetation assess ments and reduce costs during expeditions. This study presents an imagebased non-destructive method to estimate canopy attributes of wild cacao trees in two regions of the rain forest in Colombia, using digital cover photography and machine learning algorithms. Upward-looking photography at the base of each cacao tree and machine learning algorithms were used to estimate gap fraction (GF), foliage cover (FC), crown cover (CC), crown porosity $(C P)$, clumping index $(\Omega)$, and leaf area index (LAI) of the canopy cover. Here we used the cacao wild trees found on forestry plots as a case study to test the application of low-cost imagery on the extraction and analysis of canopy attributes. Canopy attributes were successfully extracted from the canopy cover imagery and provided $92 \%$ of classification accuracy for the structural attributes of the canopy. Canopy cover attributes allowed us to differentiate between canopy structures of the Amazon and Pacific rainforests sites suggesting that wild cacao trees are associated with different vegetation types. We also compare classification results for the computer extraction of canopy attributes with a digital canopy cover benchmark. We conclude that our approach was effective to quickly survey canopy features of vegetation associated with and of crop wild relatives of cacao. This study allows highly reproducible estimates of canopy attributes using cover photography and state-ofthe-art machine learning algorithms such as deep learning Convolutional Neural Networks.

Keywords: Canopy Attributes, Cover Photography, Colombia, Machine Learning, Deep Learning

\section{Introduction}

Colombia is considered as one of the main centers of diversity for crop wild relatives of cacao (González-Orozco et al. 2020). The genus Theobroma and Herrania, as well as wild species of Theobroma cacao $\mathrm{L}$., are the main taxonomic entities of cacao (Cuatrecasas 1964). They grow in remote areas of rainforests where much of its diversity is present, but accessing those regions is challenging. Studying crop wild relatives is a priority for the conservation of genetic resources (Maxted et al. 2007). Unfortunately, the available information about these crop wild relatives of high agricultural, economic, and cultural importance is limited. Accurate estimate of forest canopy structure is central for a wide range of ecological studies and applications. Because of the difficulty of direct measurements, indirect methods have been widely used. Canopy photographic methods are among the most widely used on account of their simple, fast, and costeffective procedures.

In the past, tree crown attributes have been estimated using vertical digital photography (Brown et al. 2000, Patterson et al. 2011). Digital cover photography (DCP) is a high resolution, restricted-view angle method, that provides mainly vertical sampling of the canopy (Smith et al. 2008, Chianucci et al. 2014b, 2016, Alivernini et al. 2018, Chianucci 2020) and is an emerging method to estimate canopy attributes (Chianucci \& Cutini 2012, Chianucci 2020). Accurate estimates of canopy attributes using DCP overcomes the difficulties of hemispherical photography, which are sensitive time-consuming (Chianucci et al. 2014a, Alivernini et al. 2018). Cover photographs also provide higher resolution than hemispherical photographs. In terms of image processing, machine learning algorithms have been used to estimate forest canopy imputation using Advanced Spaceborne Thermal Emission and Reflection Radiometer (ASTER) data (Shataee et al. 2012), as well as other remote sensing images (Noorian et al. 2016). For instance, canopy hemispherical photography (CHP) segmentation and gap fraction (GF) calculation were performed using deep learning neural networks (Li et al. 2020). Deep learning regression has also been used to make hemispherical photography independent of sunlight illumination conditions (Díaz et al. 2021). Here, we use upward-looking DCP (as in Chianucci et al. 2014a) rather than to image processing, which are tedious and 
downward-looking photographs because of load constraints during fieldwork in the expeditions as well as constraints in human resources.

This work addresses the issue of how to compute canopy cover properties using DCP and machine learning algorithms. Clas sification accuracy is estimated using crossvalidation and comparison to a digital cover photography benchmark (Grotti et al. 2020).

\section{Materials and methods}

\section{Study area}

The study of crop wild relatives (CWR) of cacao trees was performed in the rainforest of three Colombian departments: Caquetá, Putumayo, and Chocó (Fig. S1 on Supplementary material) between 2018 and 2019. The first cacao-BIO expedition traveled to the Caguán and upper Caquetá rivers in Caquetá and Putumayo depart ments, where five parcels surrounding a wild Theobroma tree were examined across different landscapes (flooded, firm ground, and riverbanks). Later, the second expedition took place in La Victoria municipality part of the Canton de San Pablo (Chocó department) where three parcels around a wild cacao tree in the rain forest were examined. The expedition collected a total of eight upward-looking DCPs, one for each parcel.

\section{Estimation of canopy properties}

A key point to estimate canopy attributes is to separate canopy large gaps from normal size gaps (Smith et al. 2008, Chianucci \& Cutini 2012, Chianucci et al. 2014a, Alivernini et al. 2018). One of the latest tools provides a free Python library called "Can opy Cover" (CaCo - https://github.com/aliv ernini/caco) that performs a segmentation of the canopy and its gaps, as well as a seg-

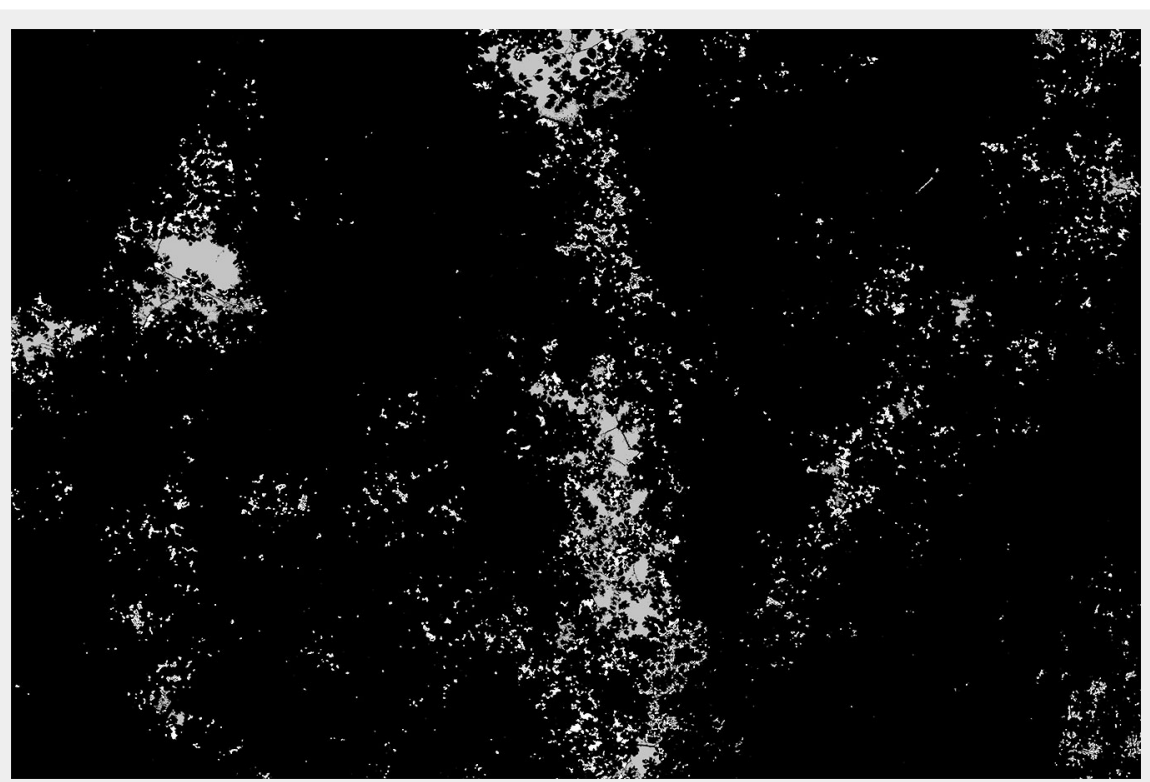

Fig. 1 - Canopy large and small gaps (black: canopy; white: small gaps; gray: large gaps) in the rain forest in Colombia. mentation of large gaps and normal size gaps using statistical methods, from DCP (Alivernini et al. 2018 - see Fig. 1 for an illustration of the canopy, small and large gaps). CaCo does not currently provide all the canopy attributes computed here, since it centers more on providing statistics of the gaps found. In this work, six machine learning algorithms are used to classify upward-looking DCPs into the sky (gaps), leaves, and trunks: K-Nearest Neighbors (KNN - Altman 1992), Support Vector Machines (SVM - Chen 2009), Random Forests (RF - Ho 1995), Extreme Gradient Boost (XGBoost - Cheng \& Guestrin 2016), Multilayer Perceptron (MLP - Rumelhart et al. 1986) and deep learning Convolutional Neural Networks (CNN - Krizhevsky et al. 2017). The algorithm in $\mathrm{CaCo}$ that statistically separates large gaps from normal size canopy gaps was used here, based on the sky class, detected using supervised machine learning algorithms. The Python code used here is available in GitHub (https://github.com/julioduarte2020/Canop yCover), where a modified version of $\mathrm{CaCo}$ is included that estimates all canopy attributes. The innovation of this work is the use of DCP and supervised machine learning algorithms to classify the images and then estimate the canopy attributes.

Samples of the sky, trunk, and leaves were selected on each DCP image using the free software MultiSpec (https://eng ineering.purdue.edu/ biehl/MultiSpec/) to form the training data. Five-fold cross-validation was used to estimate the performance of each classifier, i.e., the samples selected are randomly split into training samples (80\%) and testing samples (20\%) five times, covering the training data. The performance of each classifier was measured in terms of classification accuracy, sensitivity, and specificity. KNN, SVM, and RF classifiers were implemented in python using the Sklearn library. XGBoost was implemented in python using the XGBoost library. MLP and CNN classifiers were implemented in python using the Keras library with Tensorflow under the hood. Encouraging results were obtained by setting 5 neighbors and leaf size of 100 for the KNN classifier. The linear SVM classifier was used with default settings. As for RF classifiers, best results were obtained using 100 estimators and default settings. Regarding XGBoost, encouraging results were obtained using 100 estimators and trees as the booster. For the MLP classifier, the best results were obtained using two dense layers of size three with batch normalization (Ioffe \& Szegedy 2015) and Relu activation (Glorot et al. 2011). Also, encouraging results were obtained for the CNN classifier using a sliding window of size 9 pixels around each pixel, a first convolutional layer with a kernel of size 3 and 20 filters, batch normalization and dropout layer (Srivastava et al. 2014) of 0.2; a second convolutional layer with a kernel of size 5 and 40 filters, batch normalization, dropout of 0.2 and max-pooling of size $2 \times 2$. After the two previous convolutional layers, a flatten layer is added, followed by two MLP layers of size half of the input of the previous layer, batch normalization, dropout of 0.2 , and Relu activation.

With each DCP image classified into trunk, leaves, and sky, the following canopy attributes can be estimated (Alivernini et al. 2018 - eqn. 1 to eqn. 5):

$$
\begin{aligned}
& F C=1-\frac{g_{T}}{p_{C}}=\frac{L}{p_{C}} \\
& C C=1-\frac{g_{L}}{p_{C}} \\
& C P=1-\frac{F C}{C C} \\
& \Omega=\frac{(1-C P) \ln (1-F C)}{F C \ln (C P)} \\
& L A I=-C C \frac{\ln C P}{k}
\end{aligned}
$$

where $F C$ is the foliage coverage, $C C$ is the crown cover, $C P$ is the crown porosity, $\Omega$ is the clumping index, $L A l$ is the leaf area index; $g_{T}$ is the total number of pixels of gaps (sky); $L$ is the number of pixels of all leaves; $p_{c}$ is the number of pixels in the image minus the number of pixels of the trunk, i.e., the number of pixels of the canopy; $g_{T} / p_{C}=$ GF is the total gap fraction; $g_{L}$ is the number of pixels of large gaps, estimated as those gaps which size is larger than one standard deviation above the mean of all gaps (Alivernini et al. 2018); and $k$ is the coefficient of extinction, which is assumed to be 0.5 as in Alivernini et al. (2018).

\section{Benchmark}

Besides cross-validation classification accuracy and accuracy with respect to the 
training data, we also tested the best two classifiers: CNN and RF as well as CaCo using a digital canopy cover benchmark (Grotti et al. 2020) that consists of 315 DCP images distributed on seven test sites (45 images on each test site), taken in a hemispherical way (zenith angles between $2.5^{\circ}$ and $72.5^{\circ}$ at intervals of $5^{\circ}$ ), and using a Terrestrial Laser Scanning (TLS) to estimate the total gap fraction (GF) from 3D point data cloud available from the TLS. From these 315 images, we select 63 DCP images that are upward looking (zenith angles between $2.5^{\circ}$ and $12.5^{\circ}$ ) with their respective $G F$ and effective leaf area index $\left(L A l_{\mathrm{e}}\right)$ measures. The $\mathrm{LAl}_{\mathrm{e}}$ can be computed as (Chianucci 2020 - eqn. 6):

$$
L A I_{e}=\frac{\ln (G F)}{k}=L A I \cdot \Omega
$$

The $L A l_{\mathrm{e}}$ was computed as $\ln (G F) / k$ using the $G F$ for the benchmark and $k=0.5$ so that the $L A l_{e}$ of the benchmark corresponds to the same equations used here. The $L A l_{\mathrm{e}}$ for the DCP images was computed as $L A I \cdot \Omega$, so that there is correspondence with the eqn. 1 to eqn. 5 used here. Twenty-one images of the benchmark were chosen to select training samples for the trunk, leaves, and sky, based on the availability of those classes on each image.

\section{Results}

\section{Performance of canopy classification} algorithms

Fig. 2 shows the performance of each classifier, where CNN and RF have the best performance in terms of classification accuracy, sensitivity, and specificity.

\section{Canopy attributes}

Fig. 3 shows the classification accuracy for the three best classifiers CNN, RF, and XGBoost, on each test site. As can be seen

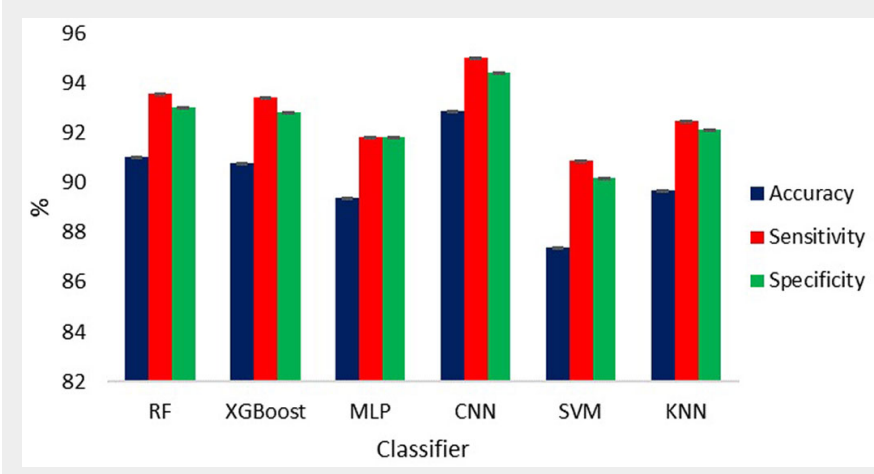

Fig. 2 - Classification performance.

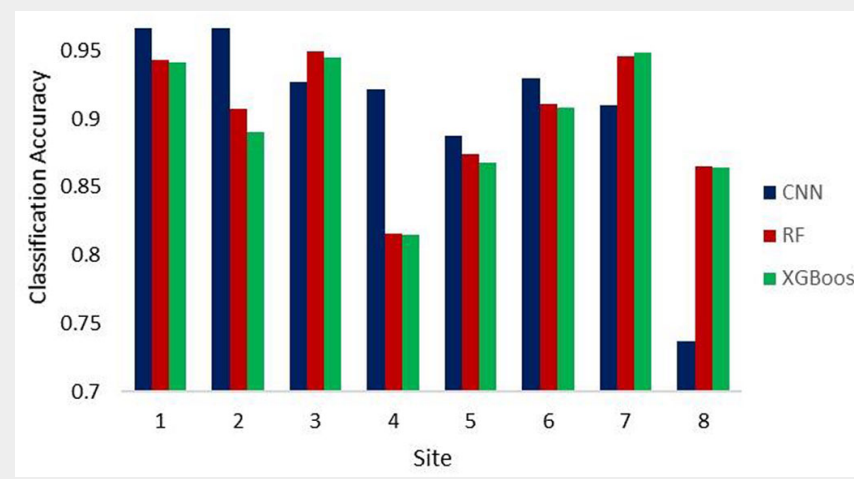

Fig. 3 - Classification accuracy on each test site. from these results, CNN seems to perform best for test sites 1, 2, 4, 5, and 6 and worst for site 8. RF and XGBoost have similar performance across all sites, being better than CNN on sites 3,7 , and 8 . In general, CNN performs well on all sites except on site 8 , where it falls behind RF and XGBoost by $13 \%$ in accuracy.

The only difference we found between the image on site 8 and the other site images is that the image on-site 8 is very sunny compared to the images on the other sites, so it is probably due to this factor that CNN does not perform well on this image.
Fig. S2-S6 (Supplementary material) show the estimated canopy attributes using CNN, RF, XGBoost, and CaCo on each test site. The first three sites correspond to Chocó and the last five sites correspond to Caquetá and Putumayo. The different classifiers showed that, in general, the Choco canopies are thicker and denser than the Caquetá and Putumayo sites.

Fig. 4 shows in the $x$-axis the TLS total gap fraction (GF) of the benchmark versus the estimated GF in the y-axis using (a) CNN, (b) RF, and (c) CaCo. From this figure, RF obtains the best $\mathrm{R}^{2}$ statistic, followed by $\mathrm{CaCo}$, and $\mathrm{CNN}$; while $\mathrm{CaCo}$ obtains the
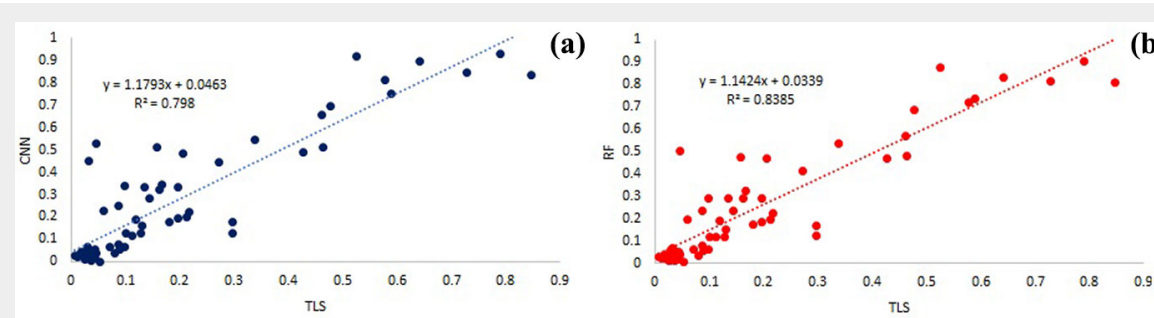

(b)

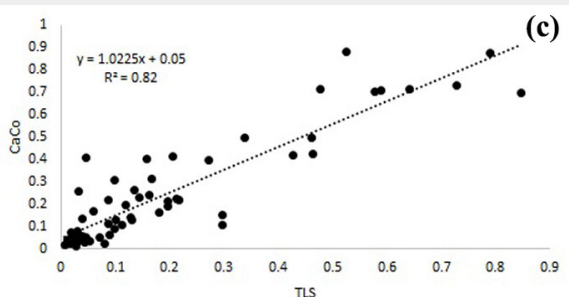

Fig. 4 - Estimated gap fraction (GF) using (a) CNN, (b) RF, and (c) CaCo vs. TLS.

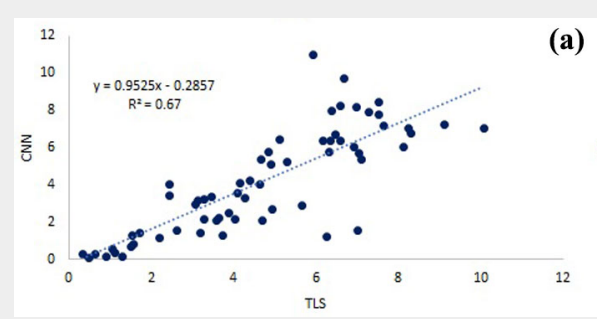

(a)

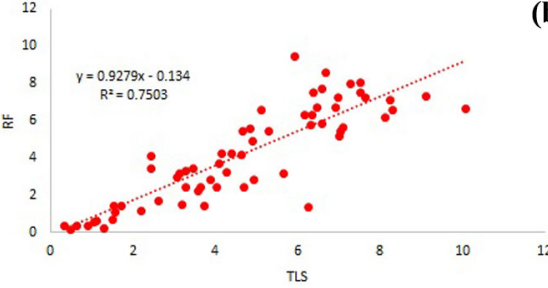

(b)

(c)

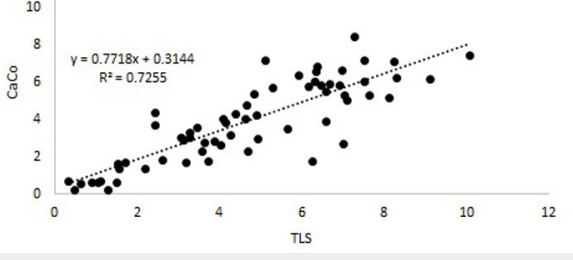

Fig. 5 - Estimated $L A l_{\mathrm{e}}$ using (a) CNN, (b) RF, and (c) CaCo vs. TLS. 
best slope. Fig. 5 shows in the $x$-axis the $L A l_{e}$ estimated using the benchmark GF versus the estimated $\mathrm{LAl}_{\mathrm{e}}$ from the images in the y-axis using (a) CNN, (b) RF and (c) $\mathrm{CaCo}$. From this figure, RF obtains the best $\mathrm{R}^{2}$ statistic, followed by $\mathrm{CaCo}$ and $\mathrm{CNN}$; while CNN obtains the best slope followed by RF and $\mathrm{CaCo}$.

\section{Discussion}

Estimated canopy cover attributes using $\mathrm{CaCo}$ tend to vary less from one site to the next, while they tend to vary more using CNN, RF and XGBoost classifiers, indicating a greater sensitivity to the varying conditions on each test site (Fig. S2-S6 in Supplementary material). The estimation of canopy cover attributes, as given in eqn. 1 to eqn. 5 , depends on a good classification of the sky, tree trunks, and leaves, as provided by the best three classifiers, i.e., $\mathrm{CNN}, \mathrm{RF}$, and XGBoost. In contrast, $\mathrm{CaCo}$ only considers two classes: sky and canopy; however, $\mathrm{CaCo}$ results are not that far away from the CNNs, RFs, and XGBoost results, showing that even though Caco was made for sky-canopy classification, it provides also good results. As a matter of fact, in some cases, $\mathrm{CaCo}$ results are closer to CNN than RF or XGBoost, also indicating a high sensitivity of the estimated canopy attributes to classification accuracy. RF and XGBoost have similar classification performance (Fig. 3) and similar estimated canopy attributes. The canopy cover benchmark estimate of the GF uses a similar skycanopy segmentation algorithm (Chianucci 2020) to CaCo (Alivernini et al. 2018), despite that CNN, and RF also obtained good prediction accuracies of $G F$ and $\mathrm{LAl}_{\mathrm{e}}$ overall.

The proposed technique can be used in agroforestry systems to estimate the canopy attributes using upward or downward DCP images, which would allow determining if for instance cacao trees are raised in a well-shaded farm, or if the programming of cultural practices such as pruning the canopy of trees is required. Canopy cover should be higher the warmer and drier the climate is, and there is a non-linear relationship between shade and yield (https://clim atesmartcocoa.guide/entry-points/shadingand-agroforestry/). The percentage of shade can be easily computed from the classification images obtained using the proposed supervised classification method.

\section{Conclusions}

A method to estimate canopy cover attributes from upward-looking DCP and machine learning algorithms have been proposed here. Given that canopy cover attributes are very sensitive to classification accuracy, it is of utmost importance to obtain good classification accuracy of the sky, tree trunks, and leaves. Deep learning convolutional neural networks provided, in general, the best classification results, compared to other well-known classification methods. Given that we compare $\mathrm{CNN}, \mathrm{RF}$ and $\mathrm{CaCo}$ against a known bench- mark and the results are satisfactory, there is confidence that the estimated canopy attributes using DCP images and machine learning algorithms are close to reality.

\section{Acknowledgments}

This work is part of the "Expedicion Colombia CacaoBIO" project carried out between AGROSAVIA and the Andes University under the special cooperation agreement of the Colombian government no. FP44842-142-2018. The Administrative Department of Science, Innovation of Colombia is acknowledged for financing the project. We thank the professional Angela Sanchez Galán (University of The Andes) for her support in the field activities.

We thank Dr. Francesco Chianucci (CREA$\mathrm{FL}$, Arezzo, Italy) for providing us with the digital canopy cover benchmark as well as the $G F$ and $L A l_{\mathrm{e}}$ measurements for those images, and for his feedback on our research through peer-review.

\section{References}

Alivernini A, Fares S, Ferrara C, Chianucci F (2018). An objective image analysis method for estimation of canopy attributes from digital cover photography. Trees - Structure and Function 32: 713-723. - doi: 10.1007/s00468-018-16663

Altman NS (1992). An introduction to kernel and nearest-neighbor nonparametric regression. American Statistician 46: 175-185. - doi: 10.1080/ 00031305.1992.10475879

Brown PL, Doley D, Keenan RJ (2000). Estimating tree crown dimensions using digital analysis of vertical photographs. Agricultural and Forest Meteorology 100: 199-212. - doi: 10.1016/S01681923(99)00138-0

Chen HF (2009). In silico log p prediction for a large data set with support vector machines, radial basis neural networks and multiple linear regression. Chemical Biology and Drug Design 74: 142-147. - doi: 10.1111/j.1747-0285.2009.0084 o.x

Cheng T, Guestrin C (2016). XGBoost: a scalable tree boosting system. In: "The Association for Computing Machinery's Special Interest Group on Knowledge Discovery and Data Mining". ACM, San Francisco, CA, USA, pp. 785-794. doi: 10.1145/2939672.2939785

Chianucci F, Cutini A (2012). Digital hemispherical photography for estimating forest canopy properties: Current controversies and opportunities. iForest - Biogeosciences and Forestry 5: 290-295. - doi: 10.3832/ifor0775-005

Chianucci F, Chiavetta U, Cutini A (2014a). The estimation of canopy attributes from digital cover photography by two different image analysis methods. iForest - Biogeosciences and Forestry 7: 255-259. - doi: 10.3832/iforo939-007

Chianucci F, Cutini A, Corona P, Puletti N (2014b). Estimation of leaf area index in understory deciduous trees using digital photography. Agricultural and Forest Meteorology 198: 259-264. doi: 10.1016/j.agrformet.2014.09.001

Chianucci F, Disperati L, Guzzi D, Bianchini D, Nardino V, Lastri C, Rindinella A, Corona P (2016). Estimation of canopy attributes in beech forests using true colour digital images from a small fixed-wing UAV. International Journal of Applied Earth Observation and Geoinformation 47: 60-68. - doi: 10.1016/j.jag.2015. 12.005

Chianucci F (2020). An overview of in situ digital canopy photography in forestry. Canadian Journal of Forest Research 50: 227-242. - doi: 10.1139 /cjfr-2019-0055

Cuatrecasas J (1964). Cacao and its allies: a taxonomic revision of the genus Theobroma. In: "Systematic Plant Studies". Smithsonian Institution Press, Washington, DC, USA, pp. 379614. [online] URL: http://repository.si.edu/hand le/10088/27110

Díaz GM, Negri PA, Lencinas JD (2021). Toward making canopy hemispherical photography independent of illumination conditions: a deeplearning-based approach. Agricultural and Forest Meteorology 296: 108234. - doi: 10.1016/j.agr formet.2020.108234

Glorot X, Bordes A, Bengio Y (2011). Deep sparse rectifier neural networks. In: Proceedings of the " $14^{\text {th }}$ International Conference on Artificial Intelligence and Statistics" (AISTATS). Fort Lauderdale (FL, USA) 11-13 Apr 2011. Proceedings of Machine Learning Research 15: 315-323. [online] URL: http://proceedings.mlr.press/v15/ glorot11a.html

González-Orozco CE, Galán AAS, Ramos PE, Yockteng $R$ (2020). Exploring the diversity and distribution of crop wild relatives of cacao (Theobroma cacao L.) in Colombia. Genetic Resources and Crop Evolution 67: 2071-2085. - doi: 10.1007/s10722-020-00960-1

Grotti M, Calders K, Origo N, Puletti N, Alivernini A, Ferrara C, Chianucci F (2020). An intensity, image-based method to estimate gap fraction, canopy openness and effective leaf area index from phase-shift terrestrial laser scanning. Agricultural and Forest Meteorology. 280: 107766. doi: 10.1016/j.agrformet.2019.107766

Ho TK (1995). Random decision forests. In: Proceedings of the "ICDAR - $3^{\text {rd }}$ International Conference on Document Analysis and Recognition". Montreal (QC, Canada) 14-16 Aug 1995. IEEExplore 1: 278-282. - doi: 10.1109/ICDAR.1995. 598994

loffe S, Szegedy C (2015). Batch normalization: Accelerating deep network training by reducing internal covariate shift. In: Proceedings of the "ICML 2015 - 32 nd International Conference on Machine Learning". Proceedings of Machine Learning Research 37: 448-456. [online] URL: http://proceedings.mlr.press/v37/ioffe15.html Krizhevsky A, Sutskever I, Hinton G (2017). ImageNet classification with deep convolutional neural networks. Communications of the ACM 60 (6): 84-9o. - doi: 10.1145/3065386

Li K, Huang X, Zhang J, Sun Z, Huang J, Sun C, Xie Q, Song W (2020). A new method for forest canopy hemispherical photography segmentation based on deep learning. Forests 11: 1-16. doi: $10.3390 /$ f11121366

Maxted N, Scholten M, Codd R, Ford-Lloyd B (2007). Creation and use of a national inventory of crop wild relatives. Biological Conservation 140: 142-159. - doi: 10.1016/j.biocon.2007.08.006 Noorian N, Shataee-Jouibary S, Mohammadi J (2016). Assessment of different remote sensing data for forest structural attributes estimation in the Hyrcanian forests. Forest Systems 25 (3): 
1-11. - doi: $10.5424 / \mathrm{fs} / 2016253-08682$

Patterson MF, Wiseman PE, Winn MF, Lee SM, Araman PA (2011). Effects of photographic dis tance on tree crown attributes calculated using urbancrowns image analysis software. Arboriculture and Urban Forestry 37: 173-179. - doi: 10.48044/jauf.2011.023

Rumelhart DE, Hinton GE, Williams RJ (1986). Learning internal representations by error propagation. In: "Parallel Distributed Process ing: Explorations in the Microstructure of Cog nition" (Rumelhart DE, McClelland JL, Williams RJ eds). MIT Press, Cambridge, MA, USA, vol. 1 pp. 318-362.

Shataee S, Kalbi S, Fallah A, Pelz D (2012). Forest attribute imputation using machine-learning methods and ASTER data: comparison of k-NN, SVR and random forest regression algorithms. International Journal of Remote Sensing 33: 6254-6280. - doi: 10.1080/01431161.2012.682661
Smith M-L, Anderson J, Fladeland M (2008). Forest canopy structural properties. In: "Field Measurements for Forest Carbon Monitoring" (Hoover CM ed). Springer, Dordrecht, Netherlands, pp. 179-176. - doi: 10.1007/978-1-4020-850 6-2_14

Srivastava M, Hinton G, Krizhevsky A, Sutskever I, Salakhutdinov R (2014). Dropout: a simple way to prevent neural networks from overfitting. Machine Learning Research 15: 1929-1958. [online] URL: http://www.jmlr.org/papers/vol ume15/srivastava14a/srivastava14a.pdf

\section{Supplementary Material}

Fig. S1 - Study sites of the expeditions CacaoBIO to the Choco department and Amazon region of Caqueta and Putumayo departments.
Fig. S2 - Estimated foliage cover using $\mathrm{CNN}$, $\mathrm{RF}, \mathrm{XGBoost}$, and $\mathrm{Caco}$, on each site.

Fig. S3 - Estimated Crown cover using CNN, $\mathrm{RF}, \mathrm{XGBoost}$, and $\mathrm{CaCo}$, on each site.

Fig. S4 - Estimated crown porosity using $\mathrm{CNN}, \mathrm{RF}, \mathrm{XGBoost}$, and $\mathrm{CaCo}$, on each site.

Fig. S5 - Estimated clump index using $\mathrm{CNN}$ $\mathrm{RF}, \mathrm{XGBoost}$, and $\mathrm{CaCo}$, on each site.

Fig. S6 - Estimated LAl using CNN, RF, XGBoost, and $\mathrm{CaCo}$, on each site.

Link: Duarte_3936@supplo01.pdf 